\title{
Uncertainty upon uncertainty: supportive Care for Cancer and COVID-19
}

\author{
Annie M Young ${ }^{1} \cdot$ Fredrick D. Ashbury $^{2,3,4} \cdot$ Lidia Schapira $^{5} \cdot$ Florian Scotté $^{6} \cdot$ Carla I Ripamonti $^{7} \cdot{\text { Ian N } \text { Olver }^{8}}^{8}$
}

Published online: 2 July 2020

(C) Springer-Verlag GmbH Germany, part of Springer Nature 2020

\section{Introduction}

Controlling the COVID-19 pandemic is having a significant negative impact on the lives of people with cancer. Despite some recent data on increased infection rates of severe acute respiratory syndrome coronavirus 2 (SARS-CoV-2) in people with cancer and increased mortality in people with COVID-19 and cancer (1), we struggle to apply these data in real-world oncology practice. Nonetheless, this illness is about people and we have recently been warned by the United Nations of a global mental health crisis due to COVID-19 (2). People with cancer are already uncertain about their future, which is now exacerbated by uncertainty over the risk of contracting the infection and not being able to receive the recommended cancer treatment (3); COVID-19 is also likely to have adversely impacted their social support network including family members, spouses, children and friends. Pandemics thrive on social inequalities and for disadvantaged people with cancer, with or without COVID-19, huge social, psychological

Annie M Young

annie.young@warwick.ac.uk

1 Warwick Medical School, University of Warwick, Coventry, England, UK

2 VieCure, Denver, CO, USA

3 Department of Oncology, University of Calgary, Calgary, Canada

4 Dalla Lana School of Public Health, University of Toronto, Toronto, Canada

5 Stanford University School of Medicine, California, USA

6 Interdisciplinary Cancer Course Department, Gustave Roussy Cancer, Center, Villejuif, France

7 Department of Oncology and Haematology, Fondazione IRCCS, Istituto, Nazionale dei Tumori, Milan, Italy

8 Faculty of Health and Medical Sciences, University of Adelaide, Adelaide, Australia and physical consequences may persist for many months or maybe years.

'Supportive care makes excellent cancer care possible', the strapline for the Multinational Association of Supportive Care in Cancer ("MASCC"), has never before been more relevant, for all countries at different stages of the pandemic, as increased stressors threaten the wellbeing of people with cancer, their caregivers and healthcare professionals. People with cancer may have built resilience to cope with its uncertainty; however, this resilience may be overwhelmed by competing fears for self and loved ones and by the variability of available psychosocial support from cancer institutions, cancer charities and global aid organizations.

In this editorial, we focus on the multi-pronged psychosocial ramifications of COVID-19 for people with cancer and their caregivers and suggest diverse integrated support mechanisms to mitigate the challenges.

\section{The challenge}

The COVID-19 pandemic, understandably, has caused anxiety. A survey of people $(n=1074)$ mostly from Hubei province, reported a higher rate of anxiety, depression, harmful alcohol use, and lower mental wellbeing during the pandemic (4). For people with cancer and their caregivers, this distress is likely to be amplified by:

Avoiding their general practitioners for signs and symptoms of cancer for fear of contracting the infection.

Having their cancer treatment delayed, cancelled or changed because of prioritization of COVID-19 cases and an attendant temporary suspension of favoured models of shared decision-making. Delaying surgery, radiotherapy and chemotherapy causes distress about potential negative outcomes (e.g. delaying cancer surgeries for three months is predicted to account for almost 5000 excess deaths in the UK) (5). 
Knowing of their vulnerability and the importance of avoiding infection e.g., fear of contracting COVID-19 and dying more quickly than from cancer.

Complicated grief reactions of families unable to say "goodbye" to dying patients with cancer because of "no visitor' institutional policies

\section{What can be done?}

Each country or regional authority has a duty of care to not only increase public awareness of signs and symptoms of cancer during and after COVID-19 but also to provide pragmatic advice of how to safely consult with the most appropriate healthcare practitioner. The engagement and education of people to safely participate in routine screening and other checks throughout the cancer care continuum, during and post-COVID, are crucial to improve outcomes.

Specifically, normalizing anxiety may help and adapting to the new reality with mindfulness, compassion and valuebased approaches is beneficial (6). Usual self-care advice on healthy strategies may be offered by healthcare practitioners good sleep habits, good nutrition and exercise. Nevertheless, such approaches are difficult during crises and patients may require referral to mental health services. Some solutions are simpler; we can seek to make the environment more welcoming and recognizing the individual patient through small gestures e.g. radiation oncologists play the patient's favourite song and have altered the landscape of the radiotherapy room during COVID-19 (7).

A screening tool of 'fear of COVID-19', developed in Iran and validated in the Italian population (8), may be used to individualize psychological care. In Paris, telehealth has allowed self-reported monitoring of cancer patients with COVID-19. Nurse navigators regularly assess and address patients' specific cancer and COVID-19 concerns, comorbidities and social conditions (9). Telephone and video calls are now common to facilitate patient consultations and can serve to provide people with cancer and their caregivers customized information to reduce their anxiety (10). The pandemic also provides new urgency to have frank conversations with patients about their preferences for end-of-life care and the worries they may have about surviving hospitalization.

\section{Social challenges}

There are benefits as well as challenges with the use of social media for information on COVID-19. Self-restraint in the choice and use of social media, is prudent. Clearly, access to accurate information on the COVID-19 crisis is essential for all. Nevertheless, many governments hide behind the crisis to restrict freedom of the press.

Socio-economic and socio-political factors globally hugely influence psychological morbidity from COVID-19; cancer patients' fears are compounded by poverty (11) and living with cancer in conflict settings (12).

Prolonged unmonitored quarantine, social distancing, stay at home and avoiding physical contact, can have heavy lasting social repercussions (13). Socializing and sharing experiences is known to reduce anxiety and depression (14); this is not possible in the COVID era and it is unclear if and how the post-COVID era will differ.

With COVID-19, there is a greater possibility of becoming unemployed, and in some countries, losing health insurance benefit coverage. The consequences of self-isolation or quarantine for financially at risk patients may lead to despair (15). Patients in financial difficulties should be encouraged to contact social workers or known support networks for expert advice. Telehealth may also reduce the feeling of being abandoned by their doctors; however, with societal 'digital divide', telehealth is not the panacea for all (10); a phonecall can help as well.

\section{Survivorship post COVID}

Cancer's life-changing experience has long lasting effects on patients, their children, spouses and family caregivers. Anxieties of cancer survivors often result from posttreatment physical symptoms (cognitive changes, insomnia or neuropathy). Moderate to severe anxiety and depression is prevalent in $9 \%$ and $17 \%$ of adult cancer survivors, respectively, 5 and 10 years post diagnosis (16). Against this background, COVID-19 may encourage some cancer survivors to interpret whether symptoms are due to COVID-19 or cancer recurrence and whether to report them, a complicated task for those striving for 'control'.

The MASCC survivorship study group has recently proposed that clinicians advance survivorship care during COVID-19 by triaging the immediate needs of cancer survivors, consider tele-survivorship care and alternate care models other than the specialist setting (17). Born of necessity, these care delivery models may eventually become our new standard. Together we need to be creative, and nimble, when designing individualized care plans that address cancer survivors' multiple psychosocial needs during COVID.

\section{Post-traumatic growth}

The diagnosis of cancer together with the threat of a novel infection with a relatively high mortality rate and for which there is no current standard therapy, has heightened the stress levels experienced by people with cancer. Nevertheless, from adversity, there are possibilities of positive transformation. The concept of post-traumatic growth (PTG) (18) can provide creative approaches for cancer care. Some PTG studies demonstrate how traumatic life events (such as a critical illness or perceived threat of disease) can also be positively 
transformational for individuals. PTG may not be a linear process as the person may shift between positive and negative states of being, but positive outcomes are more likely when the individual feels connection and greater control over their life decisions and actions.

Psychosocial and behavioural research studies in people with cancer and their caregivers have identified different coping strategies to facilitate a positive reframing of negative circumstances, including emphases on interpersonal relations, problem-solving skills and prosocial behaviours (19). Formal or informal means of peer support and interventions to facilitate cognitive reframing will become increasingly more important in the COVID and post-COVID eras of cancer care, and resources should be directed to facilitate these strategies.

Successful telehealth technologies also strive to improve social connection implemented during COVID-19 strategies and could carry on post-COVID. These have been successfully demonstrated by the adaptation of the Maggie's Cancer Caring Centre model to on-line delivery where peer support is available through online support groups, facilitated by trained healthcare professionals (20). Having a functionality that facilitates treatment plan adherence, toxicity identification and management, and bi-directional messaging can allow patients to have strong "ties" to their healthcare team and thereby help mitigate the psychosocial sequelae brought about by "distance", and perhaps yield a stronger sense of 'control' (21).

\section{Conclusion}

It is natural for people to be distressed during and post-pandemic. This distress intensifies in people at risk of or living with cancer. Although initially the cancer may have been 'forgotten' by healthcare systems whilst simultaneously struggling to protect patients from coronavirus and deliver care to those most in need, a rapid adaptation of approaches, including psychosocial interventions and telehealth to provide patient-focused support, have been evident. Simply put, cancer programmes must engage their patients: keep in touch (inperson when possible and virtually when physical proximity is precluded), listen, hear, look out for those most at risk, reassure according to the patient's values and preferences, monitor and triage psychosocial needs.

Post-COVID-19, we can expect to see ongoing economic crises and this challenge will be even more profound in low resource communities. We can expect 'new norms' of behaviour regardless of venue, and most clearly in healthcare settings. As we await a vaccine for COVID-19 and better therapeutic approaches, action is needed now. Furthermore, it is critical that clinicians and the public are given access to expert-based usable resources that encourage information exchange and engagement, and foster wellbeing. A comprehensive and integrated strategy is essential to ensure that people with cancer are given the access, care and support they need, as do their care teams, a topic for a future paper.

This editorial represents a call to action for healthcare professionals, policymakers and patient groups to acknowledge and include social and psychological factors as risks resulting from the COVID-19 pandemic. This pandemic has highlighted stark inequalities at the societal, institutional and community levels. The need for research, understanding, interventions and action is critical.

\section{References}

1. Poortmans PM, Guarneri V, Cardoso MJ (2020) Cancer and COVID-19: what do we really know? Lancet. 395(10241):1884 1885

2. United_Nations;. UN leads call to protect most vulnerable from mental health crisis during and after COVID-192020 accessed 1st June 2020. Available from: https://news.un.org/en/story/2020/05/ $1063882 \mathrm{~s}$

3. Yang G, Zhang H, Yang Y (2020) Challenges and countermeasures of integrative Cancer therapy in the epidemic of COVID-19. Integr Cancer Ther 19:1534735420912811

4. Ahmed MZ, Ahmed O, Aibao Z, Hanbin S, Siyu L, Ahmad A (2020) Epidemic of COVID-19 in China and associated psychological problems. Asian J Psychiatr 51:102092

5. Sud A, Jones M, Broggio J, Loveday C, Torr B, Garrett A, Nicol DL, Jhanji S, Boyce SA, Gronthoud F, Ward P, Handy JM, Yousaf N, Larkin J, Suh YE, Scott S, Pharoah PDP, Swanton C, Abbosh C, Williams M, Lyratzopoulos G, Houlston R, Turnbull C (2020) Collateral damage: the impact on outcomes from cancer surgery of the COVID-19 pandemic. Ann Oncol

6. Polizzi C, Lynn S, Perry A (2020) Stress and coping in the time of COVID-19: pathways to resilience and recovery. Clin Neuropsychiatry 17(2):59-62

7. Gregucci F, Caliandro M, Surgo A, Carbonara R, Bonaparte I, Fiorentino A (2020) Cancer patients in Covid-19 era: swimming against the tide. Radiother Oncol 149:109-110

8. Soraci P, Ferrari A, Abbiati FA et al (2020) Validation and psychometric evaluation of the Italian version of the fear of COVID-19 scale. Int J Ment Health Addict:1-10

9. Scotté F, Minvielle E, Mir O, André F, Barlesi F, Soria JC (2020) A patient reported outcome platform, a useful tool to improve monitoring and effective management of Covid-19-positive patients with cancer. Eur J Cancer 132:1-4

10. Elkaddoum R, Haddad FG, Eid R, et al. Telemedicine for cancer patients during COVID-19 pandemic: between threats and opportunities. Future oncology (London, England). 2020:https://doi.org/ 10.2217/fon-020-032

11. Patel JA, Nielsen FBH, Badiani AA, Assi S, Unadkat VA, Patel B, Ravindrane R, Wardle H (2020) Poverty, inequality \& COVID-19: The forgotten vulnerable. Public Health 183:110-111

12. AlWaheidi S, Sullivan R, Davies EA. Additional challenges faced by cancer patients in Gaza due to COVID-19. Ecancermedicalscience. 2020;14:ed100

13. Douglas Y. The Costs of Social Isolation: Loneliness and COVID19. Psychiatry Advisor [Internet]. April 29th, 2020 6th May 2020. Available from: https://www.psychiatryadvisor.com/home/topics/ general-psychiatry/costs-of-social-isolation-loneliness-covid19/ 
14. Santini ZI, Jose PE, York Cornwell E, Koyanagi A, Nielsen L, Hinrichsen C, Meilstrup C, Madsen KR, Koushede V (2020) Social disconnectedness, perceived isolation, and symptoms of depression and anxiety among older Americans (NSHAP): a longitudinal mediation analysis. Lancet Public Health 5(1):e62-e70

15. Lyon D (2020) COVID-19, Cancer, and financial toxicity. Oncol Nurs Forum 47(3):253-254

16. Götze H, Friedrich M, Taubenheim S, Dietz A, Lordick F, Mehnert A (2020) Depression and anxiety in long-term survivors 5 and 10 years after cancer diagnosis. Support Care Cancer 28(1):211-220

17. Chan A, Ashbury F, Fitch MI et al (2020) Cancer survivorship care during COVID-19-perspectives and recommendations from the MASCC survivorship study group. Support Care Cancer:1-4

18. Tedeschi RG, Calhoun LG (1996) The posttraumatic growth inventory: measuring the positive legacy of trauma. J Trauma Stress 9(3): $455-471$
19. Danhauer SC, Case LD, Tedeschi R, Russell G, Vishnevsky T, Triplett K, Ip EH, Avis NE (2013) Predictors of posttraumatic growth in women with breast cancer. Psychooncology. 22(12): 2676-2683

20. Howells L. When it all feels overwhelming 2020 [Available from: https://community.maggiescentres.org/blog/When-it-all-feelsoverwhelming-296

21. Chan RJ, Howell D, Lustberg MB, Mustian K, Koczwara B, Ng CC, Kim Y, Nápoles AM, Dixit N, Klemanski D, Ke Y, Toh YL, Fitch MI, Crichton M, Agarawal S, Chan A (2020) Advances and future directions in the use of mobile health in supportive cancer care: proceedings of the 2019 MASCC annual meeting symposium. Support Care Cancer

Publisher's note Springer Nature remains neutral with regard to jurisdictional claims in published maps and institutional affiliations. 